\title{
Die „Perle“ des Niederlausitzer Braunkohlenreviers Der Dresdner Architekt Georg Heinsius von Mayenburg und die Kolonie „Grube Marga“
}

\author{
von \\ MAXIMILIAN CLAUDIUS NOACK
}

Im Hinblick auf die Industrialisierung ist die Niederlausitz eine verspätete Region. Während diese in den anderen deutschen Montanrevieren, dem Ruhrgebiet, dem Saarland oder in Schlesien, spätestens im ausgehenden 18. Jahrhundert einsetzte und selbst das benachbarte mitteldeutsche Braunkohlenrevier bereits in der Mitte des 19. Jahrhunderts wesentliche wirtschaftliche Impulse empfing, blieb die Niederlausitz bis zum Beginn der Gründerjahre eine agrarisch dominierte Region. Natürlich bemühten sich auch hier der ansässige Landadel und die örtlichen Gutsherren um einen gewinnbringenden Einsatz der Ressourcen ihrer Güter, ${ }^{1}$ doch fehlte insbesondere für die vorhandene Braunkohle eine effiziente Verwendung. In den Industrierevieren an Rhein, Ruhr und Saar sowie in Schlesien gab es neben Steinkohle auch Eisen, das damit verhüttet werden konnte, und im mitteldeutschen Raum war es die Zucker- und Kaliindustrie, die sich den Braunkohlenbergbau "großgezogen"2 hatte. Erst der technologische Fortschritt machte eine sinnvolle Nutzung dieses Bodenschatzes auch in der Niederlausitz möglich. So wurde z. B. der Einsatz der Braunkohle in der Glas- und Keramikindustrie erst mit der Entwicklung des Siemensschen Regenerativgasverfahrens möglich. Ebenfalls hinderlich wirkte sich der fehlende Anschluss der Region an das deutsche Fernbahnnetz aus. Als dieser um 1870 erfolgte, gab dies den wesentlichen Impuls für eine rasante industrielle Entwicklung.

Vor allem Baustoffe und Briketts waren es, die hier produziert wurden und in den Gründerjahren eine besondere Nachfrage erfuhren. Entlang der Bahnstrecken, die nun sukzessive das junge Industrierevier erschlossen, entstanden zahlreiche Brikettfabriken, Ziegeleien und Glashütten. Häufig waren es große Unternehmen und Kapitalgesellschaften, zumeist mit Hauptsitz in Berlin, die sich nun ihre „Claims“ in der Niederlausitz absteckten. Durch das veränderte Aktienrecht und durch Insiderwissen geschah dies häufig bereits bevor die endgültige

1 Vgl. Tim S. Müller, Gosda/Niederlausitz. Landnutzungswandel einer ostelbischen Gutsherrschaft zwischen ,Ökonomischer Aufklärung' und anbrechendem Industriezeitalter (1790-1860), Münster/München/Berlin 2012.

2 Festschrift zum 50-jährigen Bestehen des Deutschen-Braunkohlen-Industrie-Vereins e. V. Halle (Saale) 1885-1935, hrsg. vom Deutschen Braunkohlen-Industrie-Verein Halle, Halle 1935, S. 313. 
Streckenführung neuer Eisenbahnlinien bekannt wurde. Eines der größten Montanunternehmen, das in jenen Jahren entstand, ist die Ilse Bergbau AG. Sie ging 1888 aus den Chemischen Fabriken Kunheim \& Co. hervor, die seit 1870 in Bückgen $^{3}$ eine Oxalsäurefabrik betrieben. Nachdem der Montanbereich des Unternehmens immer größere Bedeutung erlangte, wurde dieser 1888 als selbstständige Kapitalgesellschaft ausgegründet. Ausgestattet mit ertragreichen Braunkohlenfeldern erwarb und errichtete das junge Unternehmen in rascher Folge zahlreiche Brikettfabriken. Vielleicht unter dem Eindruck der Heyeschen Werkskolonie in Annahütte oder den Kruppschen Wohlfahrtseinrichtungen in Essen gründete auch die Ilse Bergbau AG 1895 eine eigene Fürsorgeeinrichtung für ihre Belegschaft, die Ilse-Wohlfahrtgesellschaft $\mathrm{mbH}$, die sich der Versorgung der IlseArbeiter- und Beamtenschaft mit Lebensmitteln und vor allem mit Wohnraum verschrieben hatte. Dies geschah allerdings weniger aus philanthropischer Überzeugung, als vielmehr aus wirtschaftlichem Kalkül, denn durch die Verbesserung der Lebens-, Arbeits- und Wohnbedingungen sicherte sich das Unternehmen die Leistungsfähigkeit und Loyalität ihrer Belegschaft und brachte sie zudem in eine mehrfache Abhängigkeit zum Unternehmen. Insbesondere die Werkswohnung und die mit ihr praktizierte Verbindung von Arbeits- und Mietvertrag war dabei bedeutsam, diente sie doch zum einen der Disziplinierung der Beschäftigten und behinderte zum anderen die stete Fluktuation unter den Beschäftigten der Grubenbetriebe.

Disziplinieren und binden - das waren die Hauptargumente für den Werkswohnungsbau - und zwar nicht nur in der Niederlausitz. Dennoch gab es qualitative Unterschiede: Während kleinere Unternehmen häufig nur einzelne Häuser bauten, errichteten Großunternehmen ganze Kolonien mit Versorgungseinrichtungen, Schulen und Kirchen. Natürlich schrieb das Preußische Ansiedlungsgesetz ${ }^{4}$ solche Einrichtungen vor, doch in Bezug auf Qualität und Ausführung der Bauten nahm man von staatlicher Seite keinen Einfluss, sodass z. B. eine Vielzahl von Kirchenräumen auch auf lange Sicht in billigen temporären Zwischenlösungen untergebracht wurden.

Eine bemerkenswerte Ausnahme unter den Niederlausitzer Montanunternehmen stellte die Ilse Bergbau AG dar. Bereits vor der Wende zum 20. Jahrhundert galt ihre Arbeiterfürsorge als vorbildlich, doch der große Wurf gelang dem Unternehmen 1907 mit der Errichtung von Grube und Brikettfabrik „Marga“. Verantwortlich für dieses Projekt war der damalige Generaldirektor der Ilse Bergbau AG Gottlob Schumann (Abb. 1). Selbst aus der Region stammend und in einfachen Verhältnissen aufgewachsen, arbeitete er sich über Jahrzehnte im Unternehmen

3 Seit 1946 ein Ortsteil von Großräschen, der nach 1987 weitgehend den Devastierungen für den vorrückenden Tagebau Meuro zum Opfer fiel.

4 Gesetz, betreffend die Gründung neuer Ansiedlungen in den Provinzen Ostpreußen, Westpreußen, Brandenburg, Pommern, Posen, Schlesien, Sachsen und Westfalen. Vom 10. August 1904, in: Gesetz-Sammlung für die Königlich Preußischen Staaten, Berlin 1904, Nr. 29. 
nach oben. Den Zeitgenossen galt er als „self-made-man“, 5 und er stellt innerhalb des wilhelminischen Wirtschaftssystems einen neuen Unternehmer-Typus dar: den Wirtschaftsmanager. Dieser war weder Erfinder oder Techniker, noch Eigentümer des Unternehmens, er war schlicht und einfach ein fähiger Steuermann. Im besonderen Maße zeichnet diesen Typus das Denken in großen Dimensionen und das bedachte Abwägen von Gewinnaussichten und Risiken aus. Den Prinzipien dieses „think big“ folgend, erschloss die Ilse Bergbau AG unter dem Namen Marga ${ }^{6}$ ab 1906 einen Braunkohlentagebau, der geologisch und hydrologisch als problematisch galt: das ca. 10 Meter mächtige Kohlenflöz lag unter einem bis $\mathrm{zu} 30$ Meter starken Deckgebirge. Auch die dazugehörige Brikettfabrik sprengte alle bisher bekannten Dimensionen: Waren

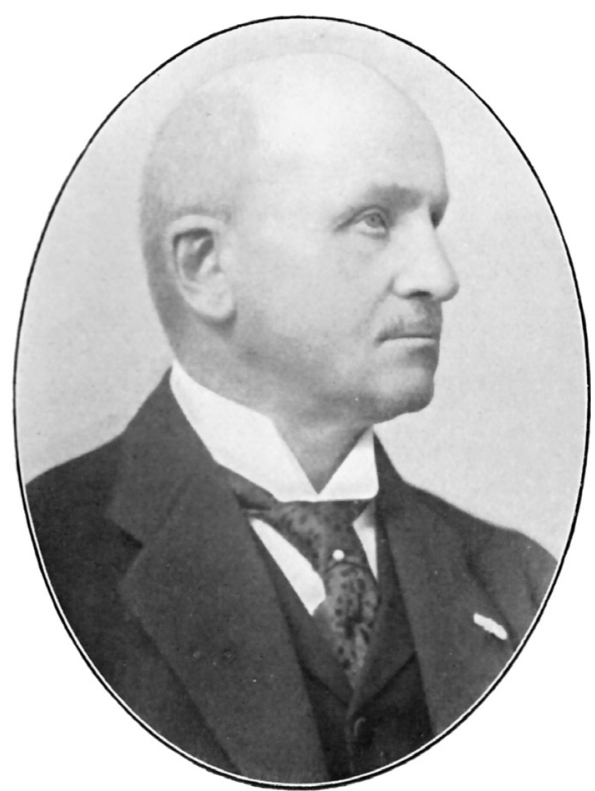

Abb. 1: Generaldirektor der Ilse Bergban AG, Kommerzienrat Dr. h. c. Gottlob Schumann, vor 1925.

Anlagen mit bis zu zehn Brikettpressen üblich, wurde die Fabrik Marga von vornherein auf eine Gesamtzahl von 38 Pressen ausgelegt (Abb. 2). Was bei den Fabrikationsanlagen zum Maßstab wurde, galt aber auch für die sozialen Einrichtungen und Nebenanlagen: So gehörten zu dem Braunkohlenwerk Marga eine moderne Kauenanlage mit Brausebädern, massive Wohnbaracken für ledige Arbeiter und eine mustergültige Kolonie (Abb. 3).

War es bisher üblich, solche Werksanlagen, inklusive der als industrielle Nebenanlagen angesehenen Wohnkolonien, durch Betriebsingenieure und ortsansässige Maurermeister planen und bauen zu lassen, wurden zur Errichtung des Werkes Marga ausgebildete Architekten hinzugezogen, die neben technischen Belangen auch ästhetische und künstlerisch-gestalterische Aspekte in ihre Planungen einbezogen. Die Ilse Bergbau AG stand damit ganz im Trend der Zeit, erinnert sei an die gestalterische Tätigkeit von Peter Behrens für die AEG in Berlin und die Gründung des Deutschen Werkbunds 1907 in München. Werbung und Produktdesign wurde nun in die Hände von Künstlern und Architekten gelegt. Das

5 Fritz-KonRAd KrÜGER, Die ökonomischen und sozialen Verhältnisse in der Braunkohlenindustrie der Niederlausitz in ihrer Entwicklung bis zur Gegenwart, Stuttgart 1911, S. 115.

6 Marga Schumann war die früh verstorbene Tochter des Generaldirektors Gottlob Schumann. Der Name Margarethe und dessen Kurzform Marga haben ihre Wurzeln im Griechischen und bedeuten „Perle“. 


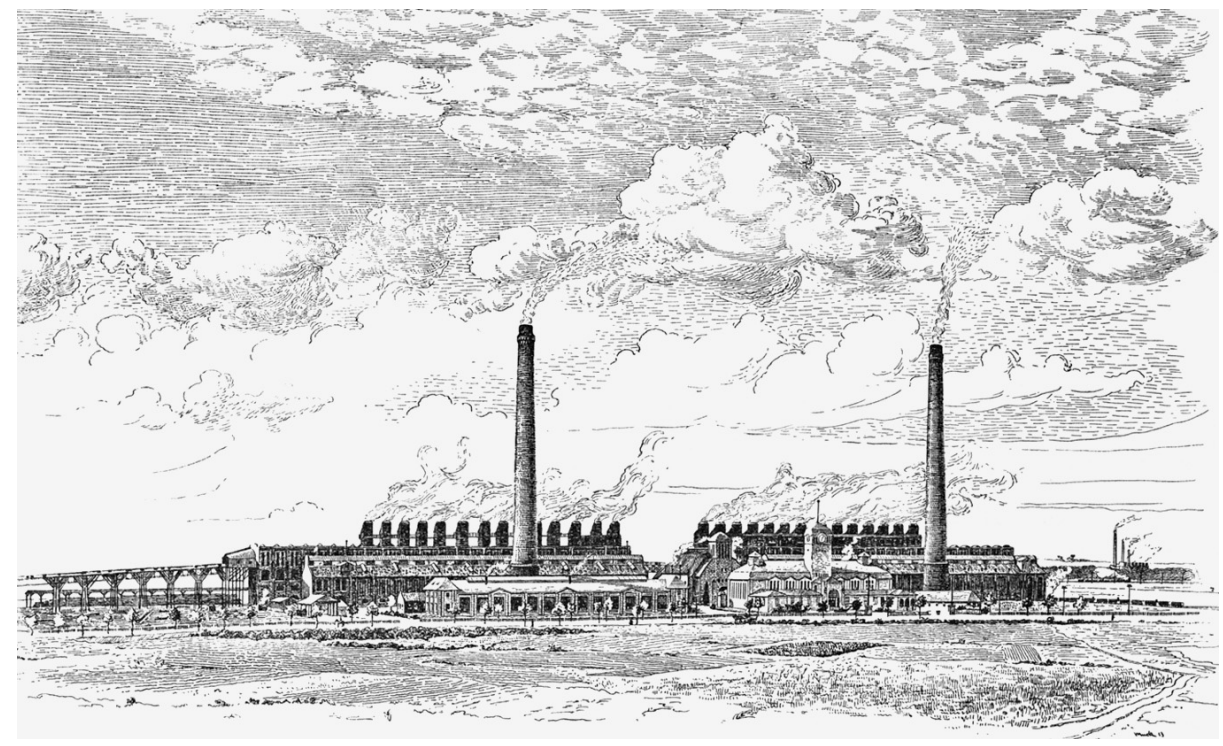

Abb. 2: Brikettfabrik Marga, erste industrielle Großanlage der Ilse Bergban AG, 1906.

erklärte Ziel war eine gute, zweckentsprechende Form, die sich im gesellschaftlichen Kontext erzieherisch auswirken sollte. In diesem Zusammenhang muss auch der nicht offene Wettbewerb für die Planungen zur Kolonie Marga gesehen werden, der an der Jahreswende 1906/1907 stattfand und an dem sich nachweislich die Architekten Wilhelm Conrad (Essen), Heidenreich \& Michel (Berlin), Georg Heinsius von Mayenburg (Dresden) und der Baurat Tieffenbach (Stralsund) beteiligten. Als Sieger aus dieser Konkurrenz ging der Dresdner Architekt von Mayenburg hervor.

Georg Heinsius von Mayenburg (Abb. 4) gehört zu der großen Zahl Dresdner Architekten, die heute weitgehend in Vergessenheit geraten sind. Die berufliche Laufbahn von Mayenburgs, der ein Schüler Ernst Gieses war, begann zu einer Zeit, als Dresden zum Zentrum der deutschen Lebensreformbewegung avancierte. Auf Kongressen und in Ausstellungen wurden hier in der ersten Dekade des 20. Jahrhunderts Konzepte zur Erneuerung in Fragen der Erziehung, der Hygiene und der Künste vorgestellt und diskutiert. Diese progressive Stimmung zog vor allem zahlreiche Künstler, Kunstgewerbler und Architekten in die Elbmetropole, darunter auch so wichtige Vertreter einer neuen Baukultur wie Martin Dülfer, Fritz Schumacher und den in Dresden omnipräsenten Hans Erlwein. Bereits die Nennung dieser Protagonisten verweist auf unterschiedliche architektonische Tendenzen und Strömungen wie Jugendstil, Traditionalismus und Protomoderne. Auch von Mayenburg experimentierte mit diesen Stilen, so finden sich in seinem Werk sowohl Einflüsse eines floralen und geometrischen Jugendstils (Schulgebäude in Pulsnitz, 1903), einer Erneuerung in der Baukunst nach englischem Vorbild (verschiedene Landhäuser in Dresden und Umgebung, zwischen 1903 und 


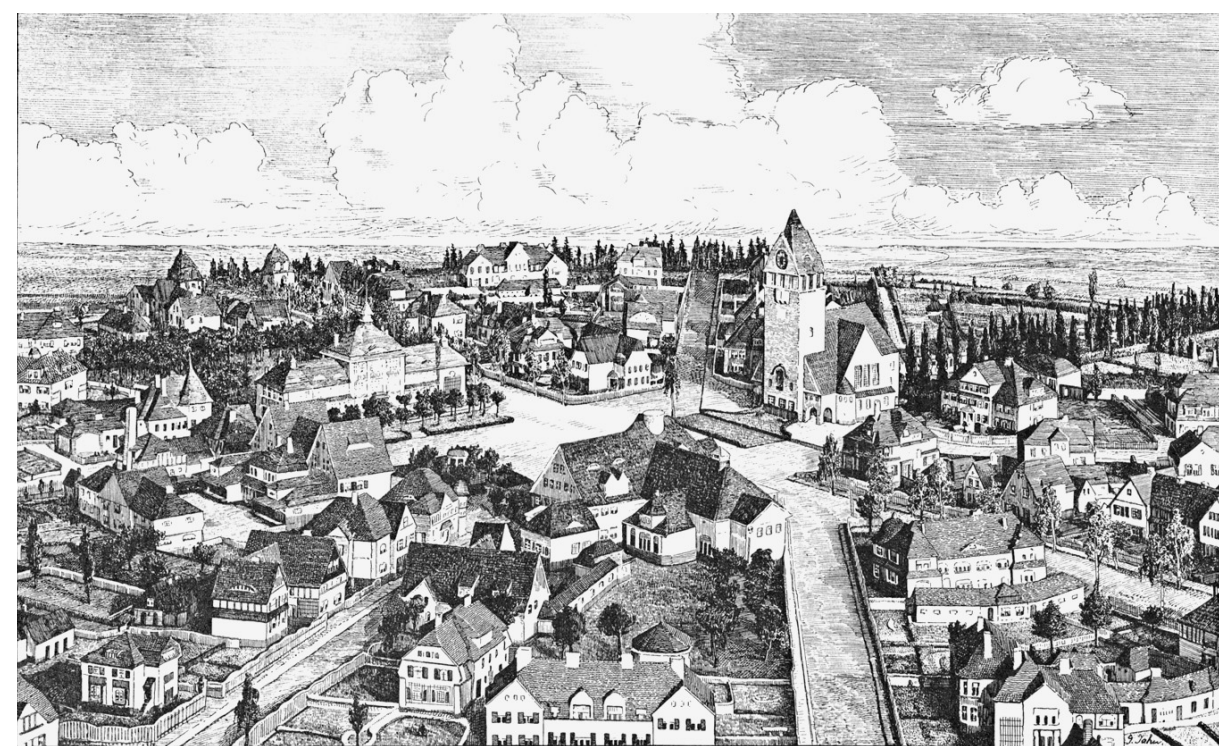

Abb. 3: Arbeiterkolonie Marga, erbaut 1907 bis 1914.

1910) und einer an barocken Gestaltungsprinzipien orientierten Architektur, die dann zur bevorzugten Ausdrucksweise des Architekten werden sollte. Von Mayenburg gehört allerdings nicht zu den Protagonisten der Dresdner Reformarchitektur, er kann lediglich als ein Vertreter aus der „zweiten Reihe“ gelten.

Auf welchem Wege von Mayenburg um 1904 in Kontakt mit der Ilse Bergbau AG bzw. ihrem Vorstand trat, ist unbekannt. Möglicherweise empfahl ihn seine Teilnahme an der Weltausstellung in St. Louis, die ihm für die Ausgestaltung eines Sekretariats eine Silbermedaille einbrachte. ${ }^{7}$ Denkbar sind aber auch Verbindungen familiärer Art, die für von Mayenburg stets von großer Bedeutung waren: Zum einen waren der Vater und ein Bruder des Architekten Bergwerksdirektoren, zum anderen bestand über einen weiteren Bruder, der als Chlorodont-Mayenburg bekannte Dresdner Apotheker und Hygieniker Ottomar Heinsius von Mayenburg, eine Verbindung in die chemische Industrie. ${ }^{8}$ Aufgrund der Gründungsge-

7 Von Mayenburg war Mitglied des Hauptvorstandes der Allgemeinen Deutschen Kunstgenossenschaft, die für die Planungen in St. Louis verantwortlich war. In dieser Funktion traf er auch auf einflussreiche Künstlerkollegen wie Peter Behrens, Bruno Möhring und Hermann Muthesius. Daneben generierte er am Rande dieser Ausstellung wohl auch wichtige Kontakte zur Wirtschaft und in die staatliche Verwaltungsaristokratie, u. a. zum Dresdner Verwaltungsdirektor und Geheimen Rat Alfred Roscher.

8 Bereits 1903/1904 erfolgte nach Plänen von Mayenburgs der Umbau der Apotheke zu Altkötzschenbroda. 1911 plante der Architekt mit dem Tautzgenhof, ebenfalls in Radebeul, eine Doppelvilla für den Generaldirektor der Chemischen Fabrik von Heyden, Richard Seifert, und den kaufmännischen Leiter, Otto Walther. In diese Zeit dürften auch Umbauten der Fabrikanlagen in Radebeul-Ost fallen, für die vermutlich ebenfalls von Mayenburg verantwortlich zeichnete. Mit den Planungen für die Seifenfabrik 


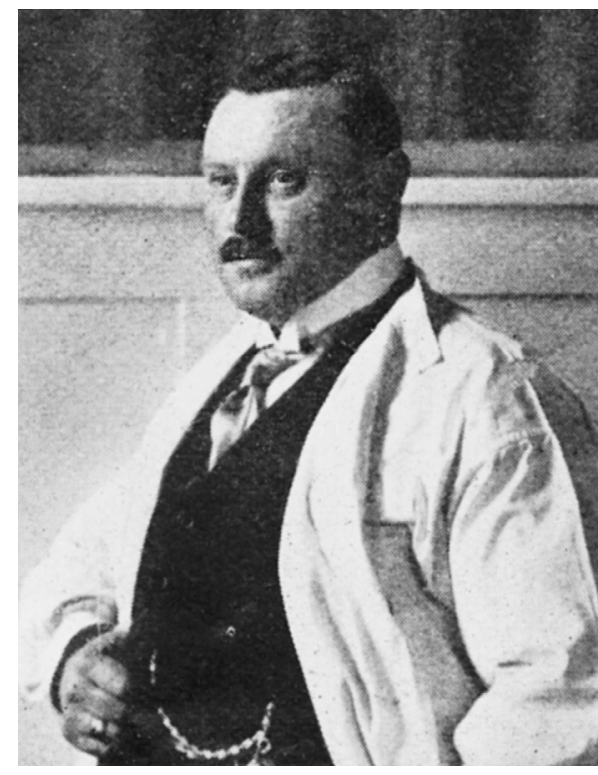

Abb. 4: Architekt Georg Heinsius von Mayenburg, Foto ca. 1912.

schichte der Ilse Bergbau AG und dem nach wie vor starken Einfluss der Familie Kunheim als deren Hauptaktionäre, scheinen beide Verbindungen möglich. Als erste Planungen von Mayenburgs für das Niederlausitzer Montanunternehmen sind Entwürfe größerer Achtund Zehnfamilienhäuser aus dem Jahre 1904 für Grube Ilse nachweisbar. Diese wurden als Wiederverwendungsprojekte auch in den anderen Kolonien des Unternehmens erbaut, so in Renate/Eva und Anna-Mathilde. Einen besonderen Auftrag erhielt der Architekt 1906, als man ihn mit den Planungen für die Erweiterung der Hauptverwaltung der Ilse Bergbau AG in Grube Ilse betraute. Dort schuf er ein Gebäude, das sich in seiner Gestalt zwischen Repräsentation und Landhausstil bewegt, das in seiner Verbindung verschiedener Materialien und Konstruktionsweisen auf die besondere Ausprägung der Reformarchitektur im Dresdner Villenbau verweist und das den Gedanken und der Formensprache der Heimatschutz-Bewegung nahesteht. Im selben Geist gestaltete von Mayenburg auch das nächste, noch umfassendere Projekt für die Ilse Bergbau AG: die Arbeiterkolonie Marga.

Lag die künstlerische Verantwortung für dieses Großprojekt bei dem Dresdner Architekten, so trug auch der erst kurz zuvor berufene Generaldirektor Gottlob Schumann Wesentliches zum Gelingen dieses Bauvorhabens bei. Nicht zufällig entstanden diese beiden Bauprojekte - Hauptverwaltung und Kolonie Marga - zu jener Zeit, als Schumann die Geschicke des Unternehmens in alleiniger Verantwortung übertragen wurden. Durch diese von der bisherigen Bautätigkeit des Unternehmens stark abweichenden Bauvorhaben signalisierte er, dass ab jetzt und in sämtlichen Belangen ein neuer Wind im Unternehmen wehte. Zugleich dokumentieren beide Bauprojekte aber auch die geistige Nähe von Architekt und Auftraggeber. ${ }^{9}$

Thierack in Finsterwalde im Jahr 1913 erhielt der Architekt erneut einen Auftrag aus der chemischen Industrie.

9 Auch Gottlob Schumann stand der zeitgenössischen Heimatschutz-Bewegung nahe, wie dessen Engagement für die Gründung des Heimatmuseums in Senftenberg (1907) und des dortigen Vereins für Heimatpflege (1909) beweist. Vgl. Ulf Јасов/ UTE Jochinke, Oasen der Moderne. Stadt- und Landschaftsgestaltungen im Lausitzer Revier (Zeitmaschine Lausitz), Dresden 2004, S. 45. 
Im Oktober 1906 suchte die Ilse Bergbau AG beim Kreisausschuss Calau um die notwendige Ansiedlungsgenehmigung nach. Das Ersuchen zeigt deutlich, dass man zum Zweck und Umfang des Bauvorhabens sehr konkrete Vorstellungen besaß, dass jedoch die gestalterische Komponente zu diesem Zeitpunkt noch unbestimmt war: Um genügende Arbeitskräfte zu erbalten, und zur Sicherung des erforderlichen Arbeiterbestandes tragen wir uns mit der Absicht, auf dem Grundstücke des von uns erworbenen Gutes Viktoriabof im Anschluss an die Guts- und Wirtschaftsgebäude eine grosse Reibe von Beamten- und Arbeiterwohnungen zu errichten. [...] Über die Grösse der Häuser, über die Gruppierung derselben und über den Umfang der Kolonie vermögen wir heute definitive Angaben noch nicht zu machen, da wir zur Erzielung möglichst zweckmässiger und praktischer Anlagen ein Preisausschreiben zu veranstalten gedenken und erst später auf Grund der erbaltenen Entwürfe die endgültige Entschliessung treffen wollen. Zunächst ist geplant, soviel Wobngebäude zu errichten, um etwa 100 Beamten- und Arbeiterfamilien unterzubringen und sollen diese Gebäude sowobl wie das erforderlich werdende Gasthaus, Kaufhaus mit der daran bängenden Bäckerei und Schlachterei im Laufe des Jabres 1907 errichtet werden. Im Laufe der späteren Jabre soll indessen die Kolonie wesentlich erweitert und auf eine Zabl von 250 bis 300 Beamten- und Arbeiterwobnungen gebracht werden. Wir haben uns gedacht, dass die Kinder der Bewohner der neu zu gründenden Ansiedlung, solange die letztere noch keinen grösseren Umfang angenommen hat, der bestebenden Schule zu Brieske zugewiesen werden, hegen aber die Absicht später eine eigene Schule für diese Ansiedlung zu errichten, sobald letztere eine entsprechende Grösse erreicht hat. Wegen der kirchlichen Versorgung bestand bei uns der Gedanke, die Bewohner der Ansiedlung der bestebenden Kirchengemeinde zu Senftenberg zuzufübren, wobin die gesamten Einwohner der Gemeinde Brieske gebören. Wir möchten auch in dieser Frage uns das Recht reservieren, in späterer Zeit vielleicht zusammen mit der alten Gemeinde Brieske unter Auspfarrung aus der Kirchgemeinde Senftenberg eine eigene Kirchgemeinde zu gründen, da die Absicht bestebt, in der Ansiedlung eine eigene Kirche zu errichten. ${ }^{10}$

Ein solch umfassendes Gebäudeprogramm, das neben den Wohnhäusern und den üblichen Versorgungseinrichtungen wie Gasthaus, Kaufhaus, Bäckerei und Fleischerei auch die Errichtung eines Schulgebäudes und einer Kirche vorsah, ist bemerkenswert. Einem solch ambitionierten architektonischen und städtebaulichen Programm galt es auch eine angemessene Form zu geben. Zu diesem Zweck lobte die Ilse Bergbau AG einen Wettbewerb unter ausgesuchten deutschen Architekten aus. Von den konkurrierenden Entwürfen hat sich nur wenig erhalten. Eine Zeichnung, die bereits auf den November 1906 datiert und die in Grube Ilse angefertigt wurde, ist vermutlich durch das werkseigene Baubüro erarbeitet worden und dokumentiert offenbar die Wünsche des Bauherrn: Sie zeigt einen zentral

10 Brandenburgisches Landeshauptarchiv Potsdam, Rep. 41 - Brieske - Nr. 51. Schreiben der Ilse Bergbau AG an den Kreisausschuss Calau vom 19. Oktober 1906. 
angeordneten Marktplatz, welcher von sämtlichen notwendigen Versorgungsbauten umstanden wird. Um diesen herum ist inmitten von Grünflächen die Wohnbebauung mit den dazugehörigen Nebengebäuden angeordnet, und die Straßen der Siedlung sollten mit Bäumen bepflanzt werden. Von den eingereichten Konkurrenzentwürfen hat sich jedoch nur eine Zeichnung erhalten, jene von Wilhelm Conrad in Essen. Sie weist genau diese Merkmale auf, weicht aber in Hinblick auf die Wohnbebauung vom Entwurf des Baubüros ab. Statt einer offenen, jedoch streng axial angelegten Blockrandbebauung mit Mehrfamilienhäusern plante Conrad - charakteristisch für das Ruhrgebiet - eine geschlossene Bebauung mit Reihenhäusern. ${ }^{11}$

Während der Vorentwurf und der Entwurf Conrads markante Gemeinsamkeiten aufweisen, bot von Mayenburg in seiner Ausführungsplanung - auch sein Konkurrenzentwurf hat sich nicht erhalten - ein ganz anderes, in sich geschlossenes Siedlungskonzept an (Abb. 5). Er entwickelte seinen Siedlungsgrundriss aus einer Spiralform heraus. Diese umfängt den zentral gelegenen, rechteckigen Marktplatz, von dem aus die Siedlung durch ein strahlenförmig angelegtes Straßensystem erschlossen wird. Strikt trennt der Architekt die beiden zentralen Bereiche der Kolonie: den Markt als öffentliches Versorgungszentrum und den ihn umgebenden halböffentlichen Bereich der Wohnbebauung. Über das „Warum“ der Spiralform ist häufig spekuliert worden: Sybille Gramlich ${ }^{12}$ sieht darin eine zukunftsorientierte Planung, die eine stete Erweiterung der Kolonie ermöglichen sollte; Paulhans Peters ${ }^{13}$ verweist auf klassische Idealstadt-Vorbilder und die Gedanken Theodor Fritzschs ${ }^{14}$ für eine „Stadt der Zukunft“ (1896); Wolfgang Joswig ${ }^{15}$ erkennt in ihr das Vorbild der Howardschen Diagramme aus dessen Buch „To-morrow“ (1898) ${ }^{16}$ und Ulf Jacob ${ }^{17}$ stellt die Bezüge zu einer zeitgenössischen romantisierenden Städtebaudiskussion her, die in der mittelalterlichen Stadtanlage ihr Ideal gefunden hatte. Ergänzt werden sollen diese möglichen Impulse um das Vorbild der barocken Planstadt Karlsruhe (ab 1715 erbaut) und um die formal ähnliche Salinenansiedlung Arc-et-Senans (1779) in der französischen FrancheComté. Wobei diesen beiden Planungen vor allem jene barocken Planungsideale zugrunde lagen, wie sie auch in der Beaux-Arts-Stadt des ausgehenden 19. Jahrhunderts eine Renaissance erfuhren. Vielleicht steht hinter dieser Form aber auch einfach nur der Gedanke, der Siedlung den eigenen Namen einzuschreiben, formal

11 Vgl. die Abb. bei Paulhans Peters, Marga: Bergarbeiter-Kolonie in der Lausitz. Entstehung, Niedergang, Sanierung, Hamburg 2002, S. 51.

12 Sybille Gramlich, Brieske. Die Kolonie Marga. Eine Arbeiterkolonie zwischen Werkssiedlungsbau und Gartenstadt, in: Brandenburgische Denkmalpflege 3 (1994), S. 85-94.

13 Peters, Marga (wie Anm. 11).

14 Theodor Fritsch, Die Stadt der Zukunft, Leipzig 1896.

15 Wolfgang Joswig, Marga. Die erste deutsche Gartenstadt, Cottbus 21999.

16 Ebenezer Howard, Garden Cities of To-morrow: Being the second edition of „tomorrow a peaceful path to real reform", London 1902.

17 Jacob/Jochinke, Oasen der Moderne (wie Anm. 9). 


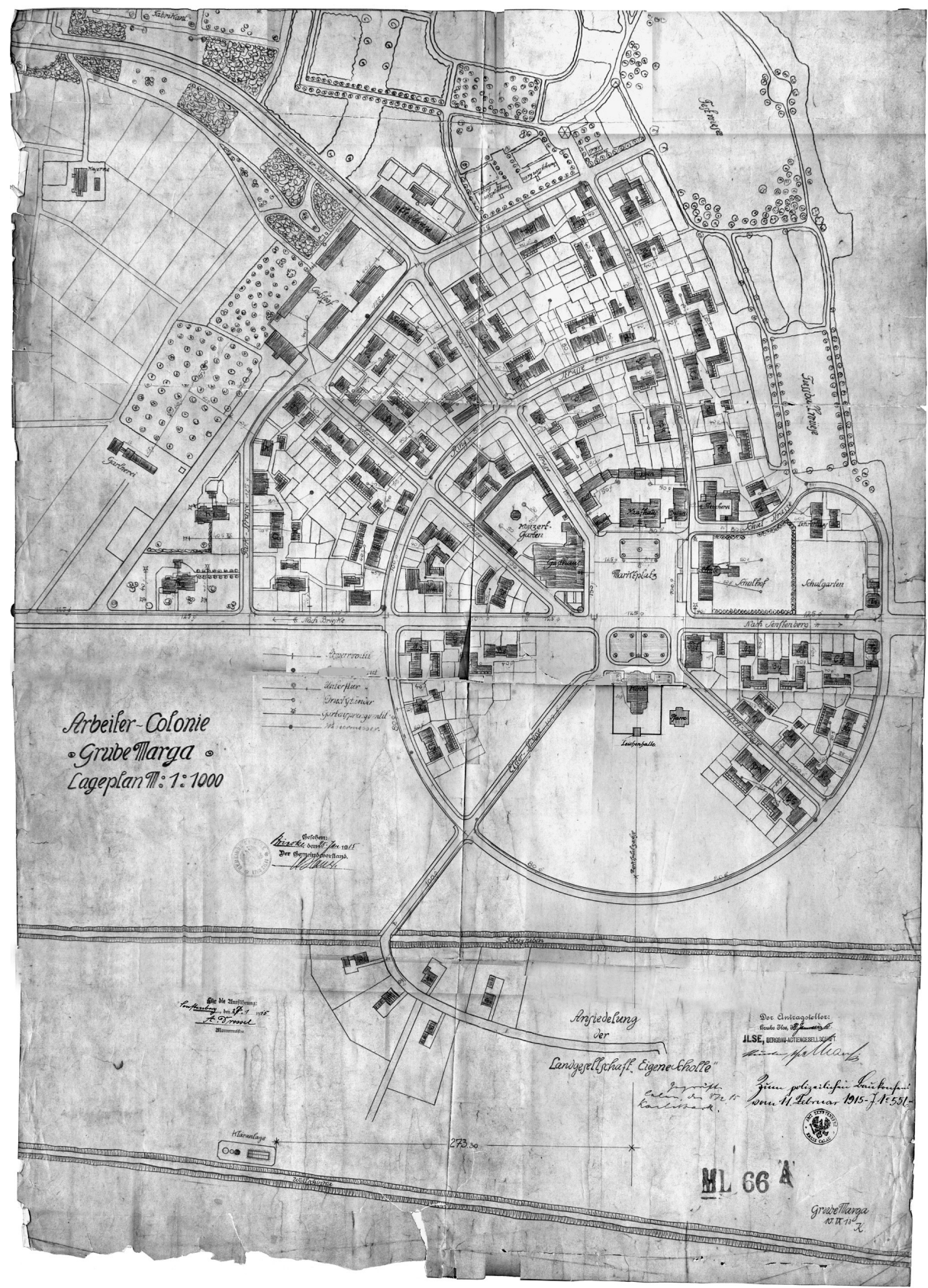

Abb. 5: Lageplan der Kolonie Marga, datiert auf den 10. September 1913. 


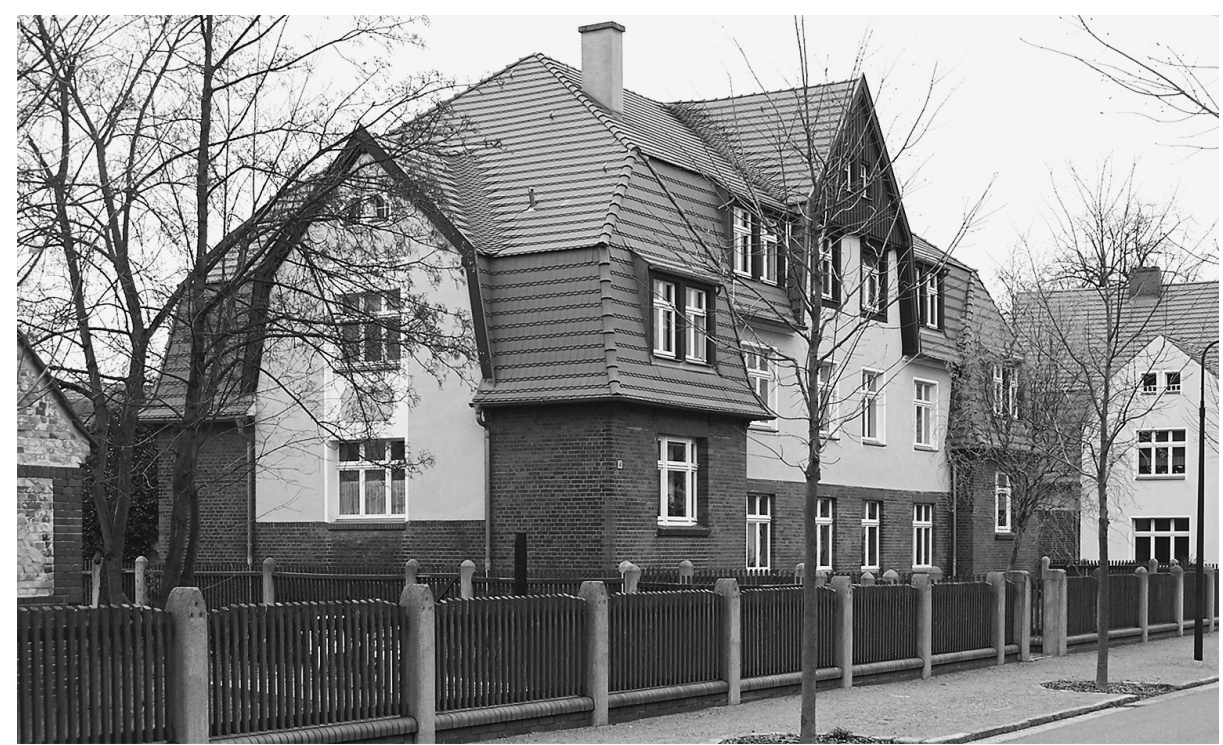

Abb. 6: Kolonie Marga, Zehnfamilienhaus, Typus B (B1-Ernst-Thälmann-Straße).

erinnert nämlich der spiralförmige Verlauf von Schul- und Ringstraße, die das Rückgrat der Siedlungsform bilden, an den Buchstaben „G“, Initial des Vornamen sowohl des Bauherrn als auch des Architekten. Nachweisbar ist diese Interpretation natürlich nicht, sie ist aber auch nicht gänzlich abwegig, zumal Schumann Werk und Kolonie bereits den Namen seiner jung verstorbenen Tochter gegeben hatte.

Grundlegend für die Planung der Kolonie scheint eine gewisse Typisierung der Wohnbauten gewesen zu sein, darauf verweisen sowohl der Vorentwurf und der Entwurf Conrads, als auch die Ausführungsplanung von Mayenburgs. Allerdings stand eine solche Typisierung dem, diesem Projekt ebenfalls immanenten, Heimatschutzgedanken konträr gegenüber, welcher Bauherr und Architekt gleichermaßen beseelte. ${ }^{18}$ Einerseits erforderten die äußeren Zwänge die Errichtung von Typenbauten, andererseits strebten Bauherr und Architekt nach einer individuell durchgebildeten Architektur, die ein gewachsenes Siedlungsganzes suggerieren sollte. Mit dem Entwurf weniger Grundformen, die in ihrem Äußeren jedoch so stark individualisiert wurden, dass nur vereinzelte Bauten einander völlig gleichen, gelang dem Dresdner Architekten diese „Quadratur des Kreises“. Für die gesamte Bebauung mit mehr als 60 Wohnhäusern entwarf von Mayenburg, dem innerbetrieblichen Status der künftigen Bewohner entsprechend, fünfzehn verschiedene Grundtypen, vom Zehnfamilienhaus mit 3-Raum- und 4-Raum-Wohnungen und einer Wohnfläche von bis zu $60 \mathrm{~m}^{2}$ bis hin zu den beiden Doppelvillen für die Werksdirektoren, die über eine Wohnfläche von jeweils $150 \mathrm{~m}^{2}$ verfügten. Es sind die großen Zehn- bzw. Achtfamilienhäuser, die die Kolonie in besonderem Maße 


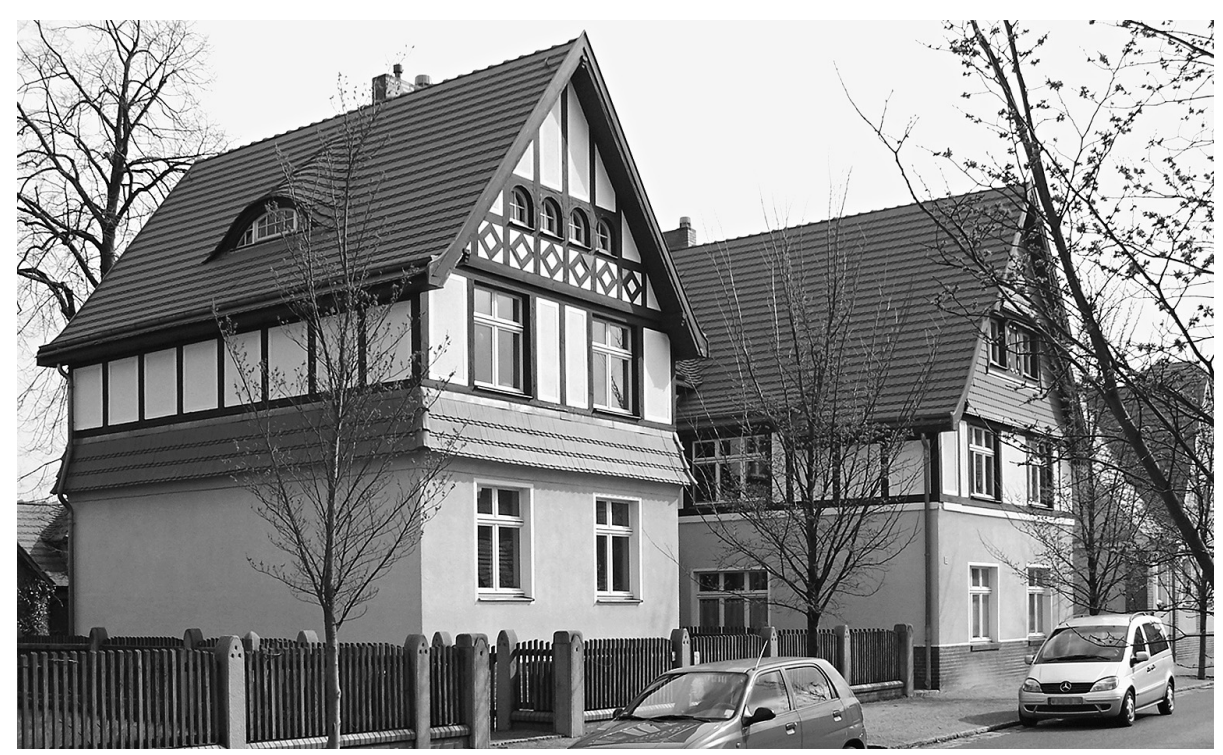

Abb. 7: Kolonie Marga, Vierfamilienhaus, Typus D (Franz-Mehring-Straße).

prägen, denn allein in den 32 Gebäuden der Typen A, B, C, L und M19 (Abb. 6) befanden sich 266 Wohnungen und damit fast drei Viertel des gesamten Wohnungsbestandes innerhalb der Kolonie. Gleichermaßen für bessergestellte Arbeiter und für niedere Beamte wurden die 13 kleineren Vierfamilienhäuser der Typen D, E, F, G und N (Abb. 7) sowie 8 Zweifamilienhäuser der Typen H und T entworfen. Für höhere und leitende Beamte errichtete die Ilse Bergbau AG hingegen die Doppelvillen des Typus K und die beiden Vierfamilienhäuser vom Typus Q. Peters bezweifelt jedoch, dass von Mayenburg mit der Entwicklung dieser Gebäudetypen eine wirkliche Typisierung und damit eine Optimierung der Erstellungskosten angestrebt hat. Für ihn steht vielmehr fest, dass die Typisierung dieser Bauten, aufgrund der minimalen Ausstattung und der rein handwerklichen Erstellung, keine wesentliche Kostenersparnis bewirkt hätte und dass diese allenfalls als Schematisierung anzusehen ist. Diesem Standpunkt soll jedoch widersprochen werden, denn zum einen wurden dadurch die Kosten für die Entwicklung der Grundrisse auf ein Viertel gesenkt, zum anderen wirkte sich dies sicher vereinfachend auf das baupolizeiliche Genehmigungsverfahren und die damit verbundenen Kosten aus. Und auch im Hinblick auf die bauliche Erstellung dürfte eine mögliche Vorfertigung von Bauelementen wie Fenster, Türen und Klappläden abseits des Bauplatzes kostenminimierend gewirkt haben.

19 Die Bezeichnung folgt der originalen Typisierung durch den Architekten. Anzumerken ist, dass diese Rückschlüsse auf den Bauablauf zulässt, sowohl in Hinblick auf die Reihenfolge ihrer Planung - A wurde vor B usw. geplant - als auch auf die Reihenfolge der Realisierung $-A_{1}$ entstand vor $A_{2}$ usw., allerdings wurde $A_{7}$ später als $B_{1}$ erbaut. 


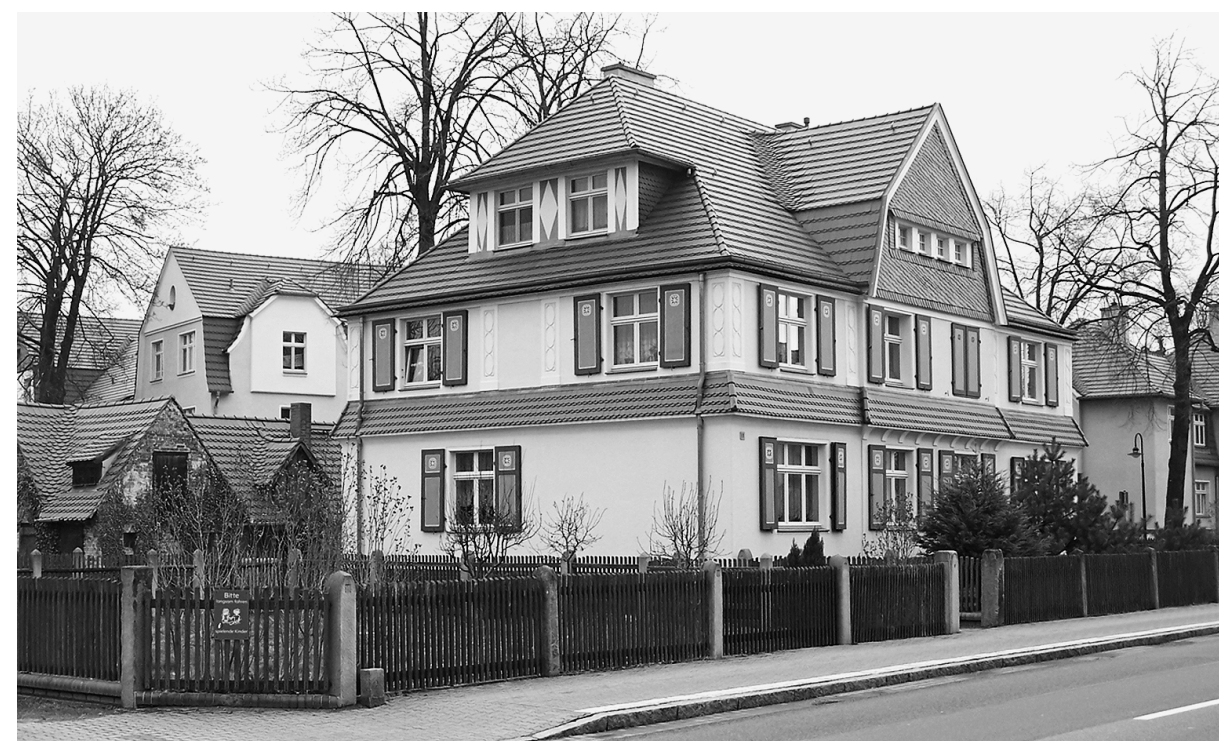

Abb. 8: Kolonie Marga, Vierfamilienhaus, Typus F (Briesker Straße).

Um den negativen Wirkungen einer solchen Typisierung entgegenzuwirken, folgte von Mayenburg bei seinen Entwürfen den Empfehlungen des Architekten und Städtebauers Karl Henrici, der auf der Hagener Konferenz ${ }^{20} 1905$ über die Gestaltung von Arbeiterkolonien referierte und der in Hinblick auf eine mögliche Uniformität und Monotonie formulierte: [...] wobei eine Abwechslung sebr leicht durch kleine Veränderungen in den Fassaden und durch die Wabl verschiedener Baumarten herbeizufübren ist. ${ }^{21}$ Aber auch in anderer Hinsicht folgte der Dresdner Architekt den Empfehlungen dieses Experten. Henrici schreibt ebendort: Was ist eine solche Arbeiterkolonie? [...] sie ist weder eine Stadt noch ein Dorf. Sie bat von beidem etwas und ist doch etwas Eigentümliches von Dorf und Stadt unterschiedliches. ${ }^{22}$ Weiter heißt es, sie sei ein neuzeitliches Gebilde ohne bistorische Vorbilder und müsse daher auch gestalterisch als solches behandelt werden. Und

20 Die sogenannte Hagener Konferenz wurde am 5. und 6. Juni 1905 durch die Centralstelle für Arbeiter-Wohlfahrt in Zusammenarbeit mit Karl Ernst Osthaus veranstaltet und befasste sich mit dem Thema „Die künstlerische Gestaltung des Arbeiter-Wohnhauses“. Die Vorträge wurden 1906 in einem Tagungsband veröffentlicht, und die Tagung erfuhr, aufgrund der Teilnahme zahlreicher einflussreicher Künstler, Architekten und Städtebauer, darunter Hermann Muthesius, Paul Schultze-Naumburg, Richard Riemerschmid, Karl Henrici und Fritz Encke, große Aufmerksamkeit in der Fachwelt. Sie kann als wichtiger Beitrag zur Gründung des Deutschen Werkbundes 1907 angesehen werden. $\mathrm{Ob}$ von Mayenburg an dieser Tagung selbst teilnahm oder ob er deren Ergebnisse aus der Fachpresse kannte, ist unklar. Sicher ist jedoch, dass die Tagung seine Planungen in gestalterischer Hinsicht nachhaltig beeinflusste.

21 Karl Henrici, Arbeiterkolonien, in: Städtebau. Zeitschrift der Deutschen Akademie für Städtebau, Reichs- und Landesplanung 1906, S. 71-76, hier S. 75. Ebd., S. 72. 


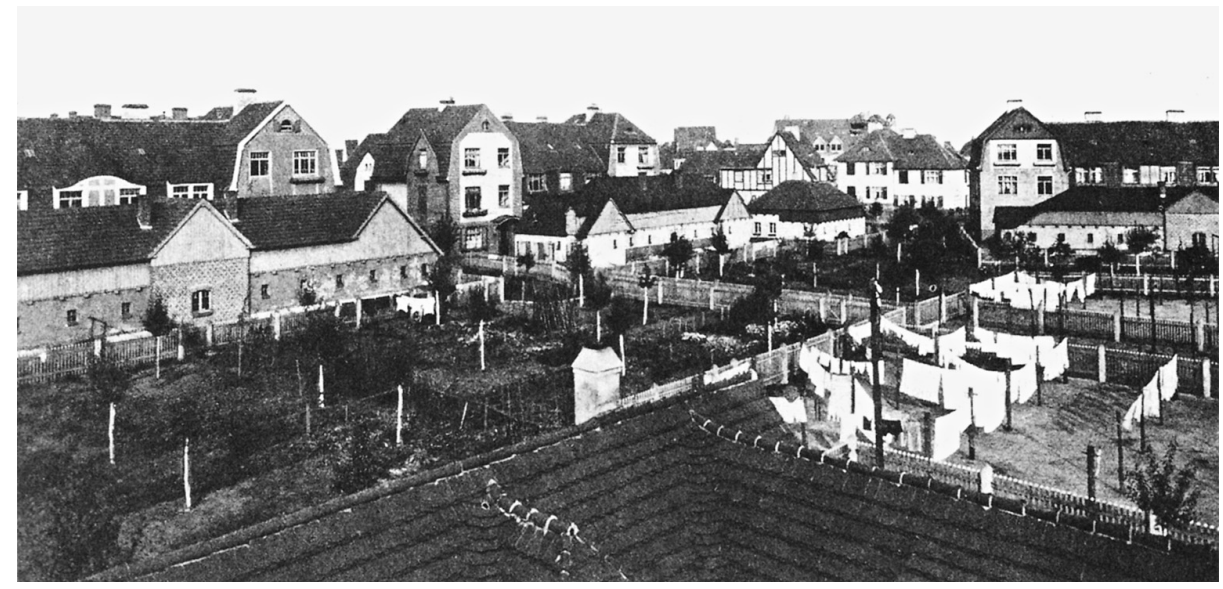

Abb. 9: Kolonie Marga, Spiel-und Wäscheplatz, ca. 1913.

Henrici konstatiert, dass aufgrund ihrer homogenen Bevölkerungsstruktur eine gewisse Uniformität unvermeidbar, diese sogar charakteristisch für eine Arbeiterkolonie sei. Für ihn war das Wohnideal innerhalb einer solchen Siedlung das kleine Einfamilienhaus mit Stall und Garten, wobei er einräumt, dass diese Wohnform nicht für alle Kolonisten die ideale sei, weshalb er das Miteinander von großen und kleinen, von städtisch und ländlich inspirierten Bauten forderte. In dieser Konsequenz beschreibt er die Arbeiterkolonien wie folgt: Dem städtischen Kerne wird sich dann nach allen Seiten der ländliche Wohnungsbau angliedern, dessen einzelne Viertel vielleicht in einem Schulbau mit Ubrtürmchen oder dergl. ibre Betonung finden mögen. ${ }^{23}$ Betrachtet man die Arbeiterkolonie Marga unter diesen Gesichtspunkten, dann findet man hier dieselben Gestaltungsmotive wieder. Große und kleine Wohngebäude wurden gleichermaßen auf den unregelmäßigen Bauarealen in lockerer, offener Blockrandbebauung angeordnet; die sich ergebenen Höfe wurden zoniert durch Funktions- und Nebengebäude, in denen sich neben Schuppen und Stallungen auch die Toiletten und Waschküchen befanden, sowie durch die Mietergärten und die gemeinsame Spiel- und Wäschewiese; einem jeden Hauseingang wurde das ländliche Motiv des Hausbaumes zugeordnet, der Schutz und Schatten spendet und der vor jedem Haus einen halböffentlichen Kommunikationsraum schafft (Abb. 8).

Formal orientieren sich die Wohnhäuser an den Traditionen ländlicher Baukultur: Kleinere Gebäude (die Typen D und E) ähneln sächsischen Dreiseithöfen wie sie u. a. in Altkötzschenbroda (Radebeul-West) noch heute zu finden sind, größere Gebäude (die Typen A, B und C) formt von Mayenburg nach dem Vorbild schlichter ländlicher Adelssitze des sächsischen Barock. Des Weiteren gestaltet er Bauten (Typus L und M, sowie einzelne Bauten des Typus C), die sich in ihrer Gestalt deutlich an das, in jener Zeit stark propagierte „englische Landhaus“ wie 


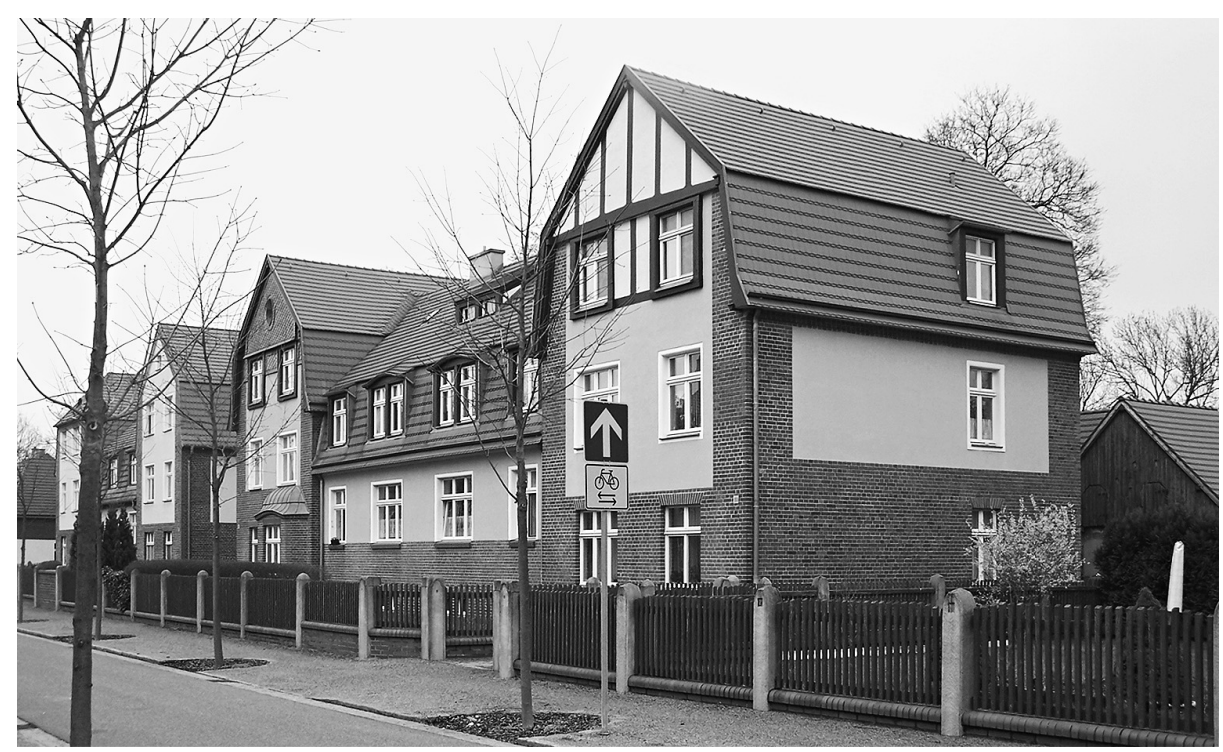

Abb. 10: Kolonie Marga, Zehnfamilienhaus Typus A (A1 - Ernst-Thälmann-Straße), Split-Level: Gut erkennbar ist der Niveaunnterschied der Fenster im Mittelteil gegenüber den Seitenbereichen der Fassade.

es durch Hermann Muthesius nach Deutschland vermittelt wurde, anlehnen, bzw. er schuf Gebäude (Typus F und G), die er dem Gestaltungskanon eines unspezifischen Biedermeiers entlehnte (Abb. 9). Immer ist aber das ländliche Motiv die Grundlage der Gestaltung. Es ist bemerkenswert, dass ihm dies selbst bei großen Gebäuden wie den Zehnfamilienhäusern gelingt. Er bedient sich dabei eines Kunstgriffs, der als „Split-Level“ bezeichnet wird: Bei dieser architektonischen Lösung befinden sich die Geschossniveaus eines Hauses bzw. einer Haushälfte nicht auf einer gemeinsamen Ebene, sondern stehen um eine halbe Etage zueinander versetzt (Abb. 10). Der Vorteil ist dabei sowohl von ästhetischer als auch sozialer Natur, denn zum einen erscheinen die Gebäude dadurch nicht so hoch, was optisch durch die weit heruntergezogenen Dächer zusätzlich unterstützt wird, zum anderen erfolgte eine stärkere soziale Trennung der einzelnen Familien, da den Wohnungen jeweils ein eigner Treppenpodest zugeordnet wird, was die ungehinderte Kommunikation von Tür zu Tür unmöglich machte. Eine solche Separierung war aber durchaus sinnvoll, zumal die Wohnungen nicht über einen eigenen Flur oder Vorraum verfügten und man nach dem Durchschreiten der Wohnungstür direkt in der Wohnküche stand.

Noch ein weiterer Architekt der Jahrhundertwende stand ideell Pate bei der Gestaltung dieser Arbeiterkolonie: Theodor Fischer. Fischer hatte sich insbesondere als Städtebauer einen Namen gemacht und mit der Textilarbeitersiedlung Gmindersdorf bei Reutlingen (1903) eine vielbeachtete Lösung für den modernen Arbeitersiedlungsbau vorgelegt. Auf von Mayenburg hat diese so inspirierend gewirkt, dass dieser Motive der Kolonie Gmindersdorf zitiert, zum einen in direk- 


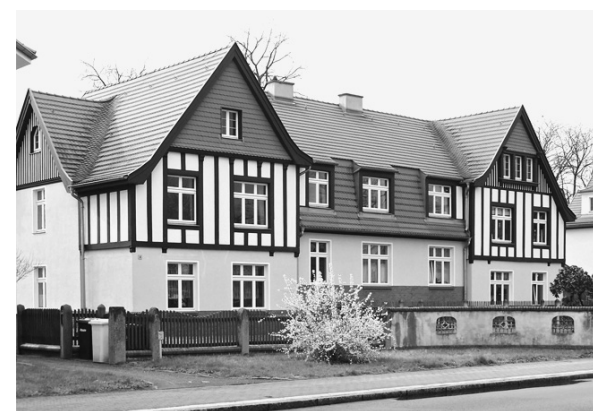

Abb. 11: Kolonie Marga, Briesker Straße.

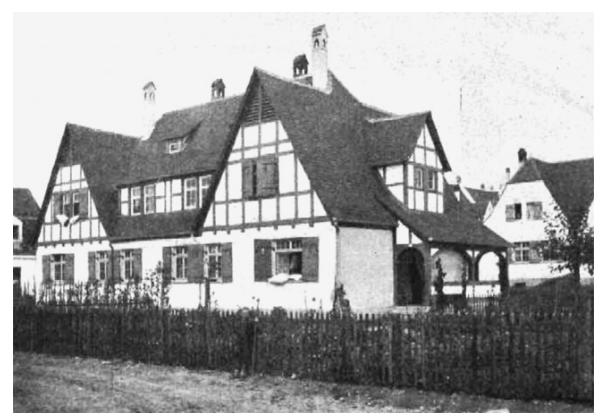

Abb. 12: Kolonie Gmindersdorf, Wilhelm-KubnStraße, erbaut ca. 1903).

ten Architekturzitaten (Abb. 11/12), zum anderen indem er sich die grundlegenden städtebaulichen Gestaltungsprinzipien Fischers zu eigen macht. ${ }^{24} \mathrm{Nach}$ Fischer galt es, zu einer funktionalen Straßenführung zurückzukehren, architektonisch und städtebaulich zwischen wichtigen und marginalen Straßen zu unterscheiden und den Übergang zwischen ländlichem Umland und städtischem Kern in einer angemessenen Form zu gestalten. Vor allem aber forderte er die Rückkehr $\mathrm{zu}$ einer [...] Gliederung nach gross und klein, nach mächtig und unbedeutend, nach Herrschen und Beherrschtwerden [...],25 ein Prinzip, das dieser als ursächlich für die wohltuende, pittoreske Wirkung historischer Stadträume beschreibt.

Auch von Mayenburg gestaltet nach diesen Prinzipien. Er gliedert seine Fassaden mittels Symmetrien und Asymmetrien, er nutzt dominante und untergeordnete Giebel, schafft Abwechslungen mittels Höhepunkten wie Ausluchten und Erkern. Und auch in städtebaulicher Hinsicht ist die Kolonie Marga nach diesen Prinzipien durchgebildet. Straßenräume werden durch unterschiedliche Bauwiche lebendig geformt und mittels Ensemblebildung wurden städtebauliche Akzente gesetzt. Im Gesamteindruck entstanden Wohnareale, die eine beachtliche Kombination des zeitgenössischen Architekturdiskurses abbilden und die die Standpunkte einer zum Teil scharf geführten Diskussion zwischen Traditionalisten wie Schultze-Naumburg und Vertretern der Protomoderne wie Fischer, Muthesius und Riemerschmid aussöhnen. Ohne direkten Bezug und ohne ein konkretes Vorbild suggerieren die Wohnstraßen der Kolonie Marga das Bild einer friedvollen, ländlichen Siedlung.

Auch bei der Gestaltung des zentral situierten Marktes orientiert sich der Dresdner Architekt deutlich an den Ausführungen Karl Henricis. Schablonengleich finden dessen Worte über eine zeitgemäße baukünstlerische Durchbildung von Arbeiterkolonien hier ihre bauliche Entsprechung: Solche Kolonie wird einen Kern bekommen, in welchem die allen Kolonisten gleichmäßig dienenden öffent-

24 Theodor Fischer, Stadterweiterungsfragen mit besonderer Rücksicht auf Stuttgart, Stuttgart 1903.

25 Ebd., S. 8. 
lichen Gebäude mit den Etagenbäusern zu ausdrucksvoller Gruppierung zusammentreten. Es wird in diesem Kern städtisches Wesen herrschen mit mebr oder weniger geschlossenen Straßen- und Platzbildern, und es wird sich dort ein Leben ein Treiben entfalten, welches die Kolonisten davon abbält, in die nächste Stadt zu pilgern, um sich zu amüsieren und das Geld los zu werden. Dem städtischen Kerne wird sich dann nach allen Seiten der ländliche Wohnungsbau angliedern [...].26 In seiner kleinstädtischen Idylle bildet der Markt den Gegenpol zu den umgebenden Wohnquartieren. Kirche, Gasthaus und Schule sowie Post, Kaufhaus, Bäckerei und Fleischerei umstehen diesen langrechteckig angelegten Platz (Abb. 13). Sie bilden ein städtebauliches Ensemble, das ebenfalls durch das Fischersche Prinzip des „Herrschens und Beherrschtwerdens“ und durch das Spiel mit Symmetrien und Asymmetrien bestimmt wird.

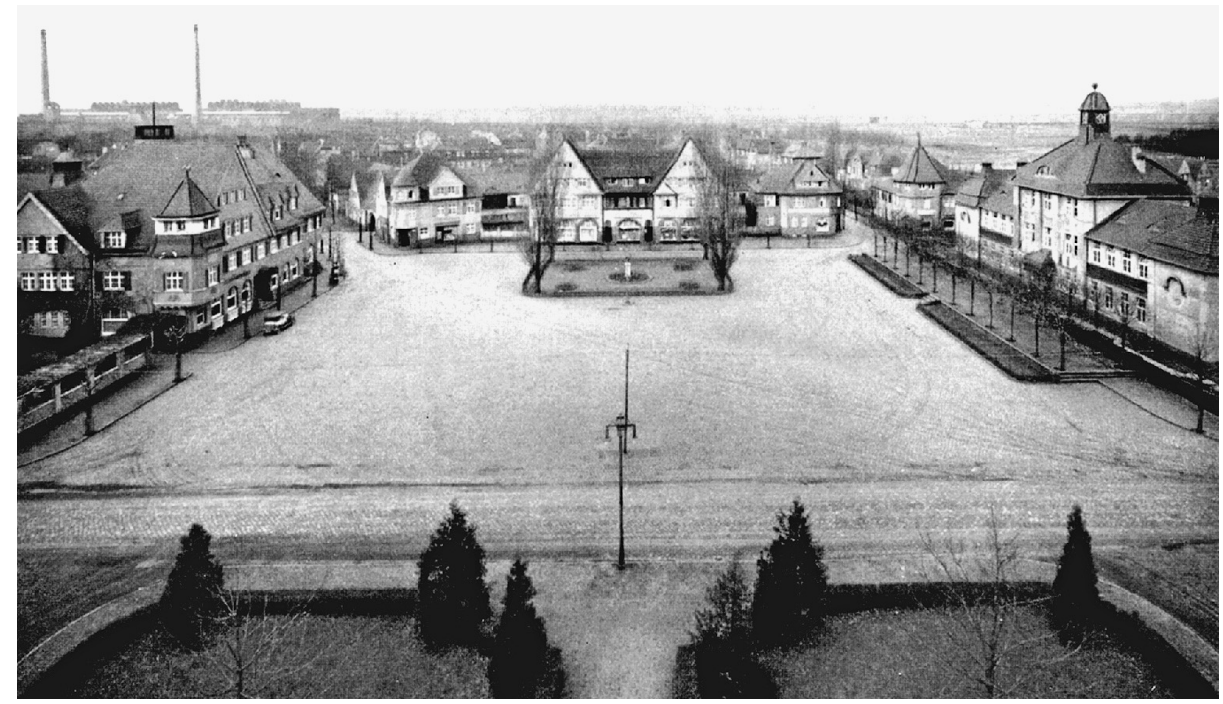

Abb. 13: Kolonie Marga, Marktplatz, um 1930.

Höhendominante am Markt und in der gesamten Kolonie ist die dem Reformator Martin Luther geweihte evangelische Kirche (Abb. 14). Dieser vis-à-vis befindet sich, mit den untereinander verknüpften Gebäuden von Post, Kaufhaus und Bäckerei sowie der separat angeordneten Fleischerei, das Konsum- und Versorgungszentrum der Kolonie. Im Kontrast zur gegenüberliegenden Kirche besitzt dieser Gebäudekomplex zwar nur eine geringe Höhe, nimmt in seiner geschlossenen Bauweise dafür aber die gesamte Flanke des Marktes ein und bildet, in seiner horizontalen Dominanz, ein Gegengewicht zur Vertikalität des Kirchengebäudes. Die beiden anderen Platzflanken werden durch die ebenfalls einander vis-à-vis angeordneten Bauten des Gasthauses und der Schule besetzt und treten vermittelnd zwischen den horizontalen und vertikalen Polen des Platzes auf.

26 Henrici, Arbeiterkolonien (wie Anm. 21), S. 74. 


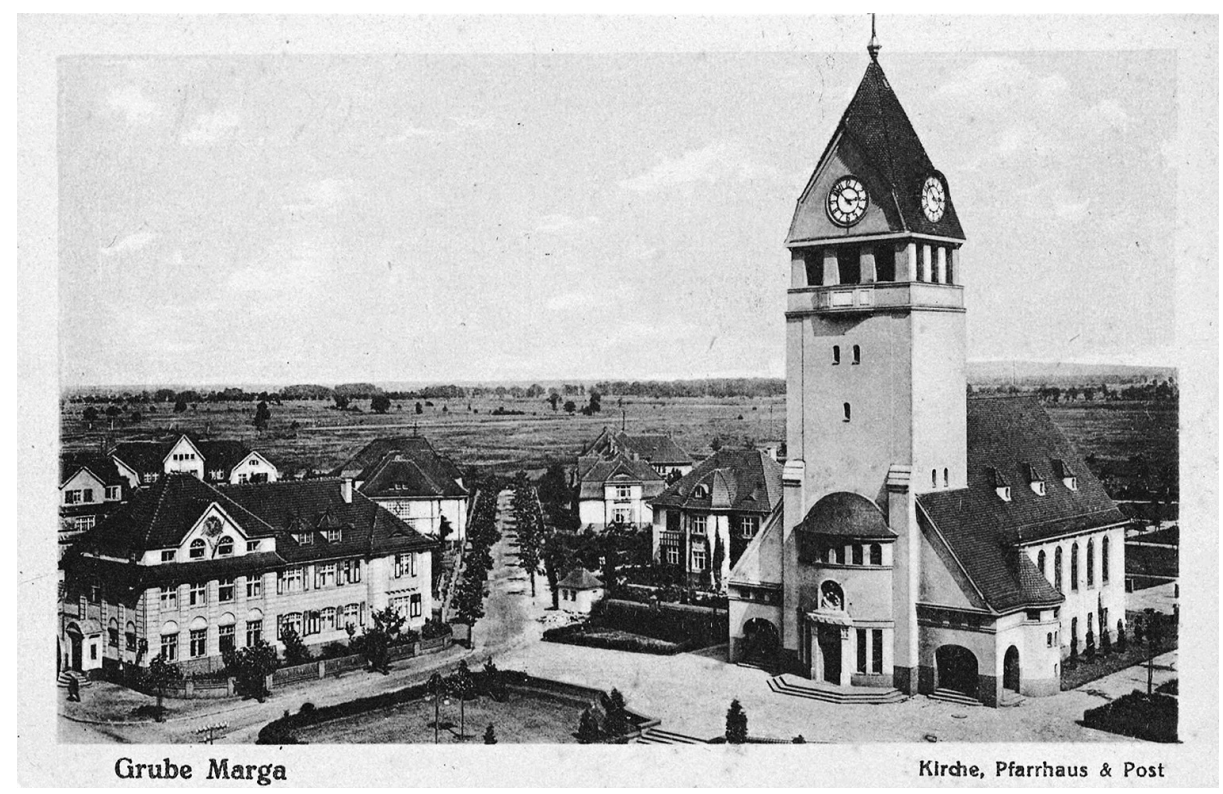

Abb. 14: Kolonie Marga, Lutherkirche, Postkarte um 1920.

Während die Gebäude, die in ihrer Funktion eher demokratischen Charakter besitzen wie Kirche, Schule und Versorgungskomplex, streng symmetrisch gestaltet wurden, ${ }^{27}$ weist das Gasthaus in seiner Fassade eine markante asymmetrische Gestaltung auf (Abb. 15). Diese bildet sowohl funktional als auch hierarchisch die innere Struktur der Gasträume im Erdgeschoss ab. Von links nach rechts befinden sich dort ein Séparée, das Restaurant, das Casino und die sogenannte Schwarze Stube. Während das Séparée der Werksleitung vorbehalten war, diente das Restaurant den Gästen des Werkes und den örtlichen Honoratioren, das Casino war für Werksbeamte reserviert und die Schwarze Stube - sie trägt diesen Namen, weil dieser Bereich auch in Arbeitskleidung betreten werden durfte - war für die Arbeiter bestimmt. Im Äußeren bilden sich diese Hierarchien durch die Verwendung verschiedener Dachformen und durch einen unterschiedlichen künstlerischen Gestaltungsaufwand ab. So markiert ein Erkertürmchen mit stilisierter Kaiserkrone als Turmzier den Bereich, den das Séparée einnimmt; ein hoher, dominanter Giebel mit einem Belvedere, reich verzierten Bleiglasfenstern und einer durchgebildeten Brustzone im Sockelbereich bildet den Funktionsbereich des Restaurants ab; das Casino schmiegt sich unter einem schlichten Walmdach eng an diesen Be-

27 Zwar erscheint der überwiegende Teil der Versorgungsbauten, jeder für sich betrachtet, dieser These zu widersprechen, doch müssen sie als Teil eines Ganzen gesehen werden, und in diesem Gesamtgefüge ergibt sich ein streng symmetrisches Bild das durch den rhythmischen Wechsel unterschiedlicher Architekturmotive geprägt wird. Von links nach rechts gliedert sich die Fassade des Versorgungskomplexes in folgender Weise: Turm, Lukarne, Giebel, breitgelagerte mehrteilige Gaube, Giebel, Lukarne, Turm. 
reich an, die künstlerische Durchbildung der Glasfenster ist hier bereits deutlich zurückgenommen; die Schwarze Stube, unter einfachem Satteldach, tritt hinter die Flucht der Hauptfassade zurück und weist nur noch im Eingangsbereich künstlerische Elemente auf, sie erscheint so nur noch als Annex zum Hauptbau. Neben diesen Gasträumen verfügte der Werksgasthof „Kaiserkrone“ über einen Hotelbetrieb in den Obergeschossen des Gebäudes und über einen Saalanbau, der dem Amüsement, aber auch der kulturellen Bildung der Koloniebewohner diente, hier fanden neben Tanzveranstaltungen auch Theateraufführungen und Konzerte statt. 28

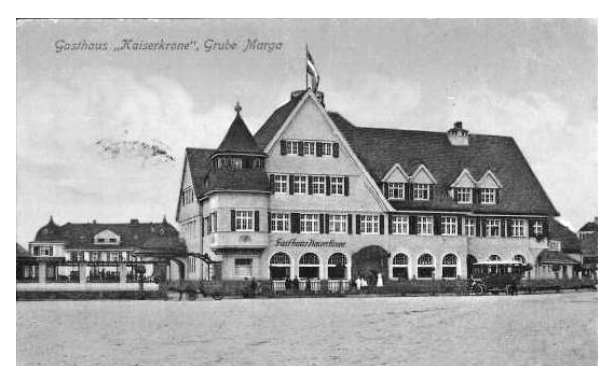

Abb. 15: Kolonie Marga, Gasthaus „Kaiserkrone", Postkarte um 1920.

Auf die weiteren Einzelbauten des Marktensembles soll hier nicht weiter eingegangen werden. Detaillierte Analysen hat der Autor im Rahmen seiner Dissertationsschrift, die 2016 erschienen ist, vorgelegt. ${ }^{29}$ Zusammenfassend kann jedoch konstatiert werden, dass die Gestaltung des Marktes einen interessanten Einblick in die baukünstlerische Arbeitsweise des Architekten von Mayenburg erlaubt. Vorbilder und Inspirationsquellen sowohl aus dem zeitgenössischen Bauschaffen als auch aus der zeitgenössischen Fachliteratur sind klar erkennbar und bestimmbar. Teils sieht sich der Architekt verschiedenen vertrauten Bauaufgaben gegenübergestellt, zu deren Bewältigung er aus persönlichen Erfahrungen schöpfen kann, teils trifft er bei der Durchbildung des Marktes auf gänzlich neue Aufgaben, die er sich zuvor erschließen muss. So ist von Mayenburg mit den Anforderungen, die der Bau eines Schulgebäudes an den Architekten stellt, durchaus vertraut, hat er doch bereits 1902 ein solches Gebäude für die Stadt Pulsnitz entworfen und war zeitgleich zum Schulprojekt in Marga auch mit dem Entwurf zweier Schulen in Riesa befasst.

Ähnlich ist die Situation, die Gestaltung gastronomischer Einrichtungen betreffend: Im Rahmen der Internationalen Hygiene-Ausstellung 1911 in Dresden hatte von Mayenburg für das dortige Vergnügungsviertel mehrere Gebäude zu gastronomischen Zwecken konzipiert. Anders verhält es sich bei Kauf- und Geschäftshäusern. Abgesehen von der Apotheke in Kötzschenbroda, ${ }^{30}$ konnte von

28 W. Joswig sieht in dem Werksgasthof mit seinem Saalanbau das Ideal des Reformgasthauses architektonisch verwirklicht, wie es zum Gesamtkonzept einer Gartenstadt gehörte. Seine Argumentation ist jedoch nicht objektiv und dient vor allem der Legitimierung der Arbeiterkolonie als Gartenstadt. Dieser Position muss daher entschieden widersprochen werden. Vgl. Joswig, Marga (wie Anm. 15), S. 46.

29 Maximilian Claudius Noack, Zwischen wilhelminischer Bedarfsarchitektur und moderater Moderne. Die Werkskolonien im Niederlausitzer Braunkohlenrevier, Diss. Dresden 2015.

30 Heute Ortsteil von Radebeul. 
Mayenburg auf diesem Gebiet kaum persönliche Erfahrungen einbringen. ${ }^{31}$ Hier orientiert er sich an gestalterischen Lösungen zeitgenössischer Architektenkollegen. Es ist das Vorbild der um 1904 in München erbauten Kaufhäuser Oberpollinger und Tietz von Heilmann \& Littmann, das der Dresdner Architekt für das Ilse-Kaufhaus in Marga in bemerkenswerter Weise adaptiert. Gleiches kann für die Gestaltung des Kirchengebäudes gelten. Auch in dieser architektonischen Teildisziplin hatte der Architekt kaum Erfahrung. Zwar hatte er während seines Studiums für seinen Lehrer Ernst Giese den Umbau der Kirche in Radibor betreut, seine Tätigkeit dort dürfte aber kaum über eine Kontrollfunktion hinausgegangen sein. Immerhin wurde ihm im Rahmen des Projektes die Innengestaltung zur selbstständigen Bearbeitung übertragen. Bei seinen Planungen für die Kirche in Marga orientiert sich von Mayenburg jedoch weniger an den, um die Jahrhundertwende zahlreichen zeitgenössischen Vorbildern. Es scheint vielmehr, als habe er sich von der Forschungsliteratur inspirieren lassen, denn seine Lutherkirche erinnert in ihrer Kubatur an eine Skizze Cornelius Gurlitts, die dieser von der Dorfkirche in Grethen bei Grimma (13. Jahrhundert) anfertigte und veröffentlichte. ${ }^{32}$ Es kann also davon ausgegangen werden, dass von Mayenburg zur Bewältigung einer Bauaufgabe ein intensives Studium der Fachliteratur betrieb bzw. die aktuellen fachlichen Diskussionen sehr genau kannte. Diverse Planungsdetails erhärten diese These: So scheinen in Marga die Gestaltungsempfehlungen Gurlitts zum Kirchenbau, die dieser für das „Handbuch der Architektur“ formulierte, treulich umgesetzt; gleiches gilt für die Empfehlungen Gustav Behnkes für „Volksschulen und niederere Schulen“ oder die Empfehlungen Robert von Neumanns, die „Gebäude für den Post-, Telegraphen- und Fernsprechdienst“ betreffend - Publikationen, die allesamt im Rahmen des „Handbuchs der Architektur“ erschienen sind. ${ }^{33}$

Unter diesem Gesichtspunkt wird man resümieren können, dass der Architekt Georg Heinsius von Mayenburg ganz gewiss mit der zeitgenössischen Diskussion um die englische Gartenstadtbewegung vertraut war, die u. a. auch in einschlägigen deutschen Fachzeitschriften für Architekten und Städtebauer geführt wurde, dass er aber keinesfalls die Errichtung einer Gartenstadt im Howardschen Sinne angestrebt hat. Während diese nämlich auf demokratischen Strukturen wie dem gemeinschaftlichen Eigentum an Grund und Boden basiert, ist die Kolonie Marga vielmehr ein konservatives, hierarchisch strukturiertes Gebilde, dessen vorrangige Aufgabe es war, das Verhältnis zwischen dem Unternehmen und dessen Arbeitern, welches zwischen Abhängigkeit und Loyalität oszillierte, zu manifestieren. Zum einen geschah dies ganz direkt, durch die Verknüpfung von Arbeits- und Mietver-

31 Die Aufträge zur Planung zweier Wohn- und Geschäftshäuser in Finsterwalde bzw. eines solchen in Annaberg erhielt von Mayenburg wohl erst nach 1910.

32 Vgl. Cornelius GurlitT, Die Dorfkirche, in: Robert Wuttke (Hg.), Sächsische Volkskunde, Dresden 1900, S. 363-381. Denkbar ist aber auch, dass von Mayenburg diese Kirche aus eigener Anschauung kannte, da dessen Geburtsort Colditz nur wenige Kilometer entfernt gelegen ist. Allerdings entspricht die Skizze Gurlitts in ihrer Reduzierung eher dem Entwurf in der Kolonie Marga.

33 Josef Durm u. a. (Hg.), Handbuch der Architektur, Stuttgart 1890-1943. 
trag, wobei eine kurzfristige Wohnungsausweisung - nach Selbstauskunft der Ilse Bergbau AG - selten bis gar nicht vorkam. ${ }^{34} \mathrm{Zum}$ anderen geschah es auf ganz subtile Weise, indem auch die Architektur den Bewohnern mit baukünstlerischen Mitteln die Hierarchisierung in Werk und Kolonie stetig vor Augen führte: Groß und klein, oben und unten, herrschen und beherrscht werden, das sind die zu diesem Zweck eingesetzten Motivpaare. Selbst die „künstlerische Ausstattung“ thematisiert diese Hierarchie in Form von drei Relief-Medaillons: Dargestellt sind dort ein Pelikan mit seinen Jungen, sich die Brust mit dem Schnabel öffnend; ein Putto mit Blütengirlande und Bienenstock sowie zwei Putti, um einen Apfelbaum tanzend (Abb. 16 bis 18). Hier wurden Motive der christlichen Ikonografie im unternehmerischen Sinne umgedeutet. So steht der Pelikan hier natürlich nicht für das Opfer Christi, sondern vielmehr für das Opfer des Arbeitgebers, der den Seinen ein solch schönes Wohnumfeld geschaffen hat; der Bienenstock steht demnach nicht für die gottgegebene Ordnung in Kloster und Kirche, sondern vielmehr für ihre Entsprechung in Werk und Kolonie sowie für den Bienenfleiß, den der Arbeitgeber als Loyalitätsbekundung erwartet, und auch der Tanz um den Apfelbaum steht nicht für das himmlische Paradies, sondern vielmehr für das Arbeiterparadies, in dem die Koloniebewohner leben dürfen.
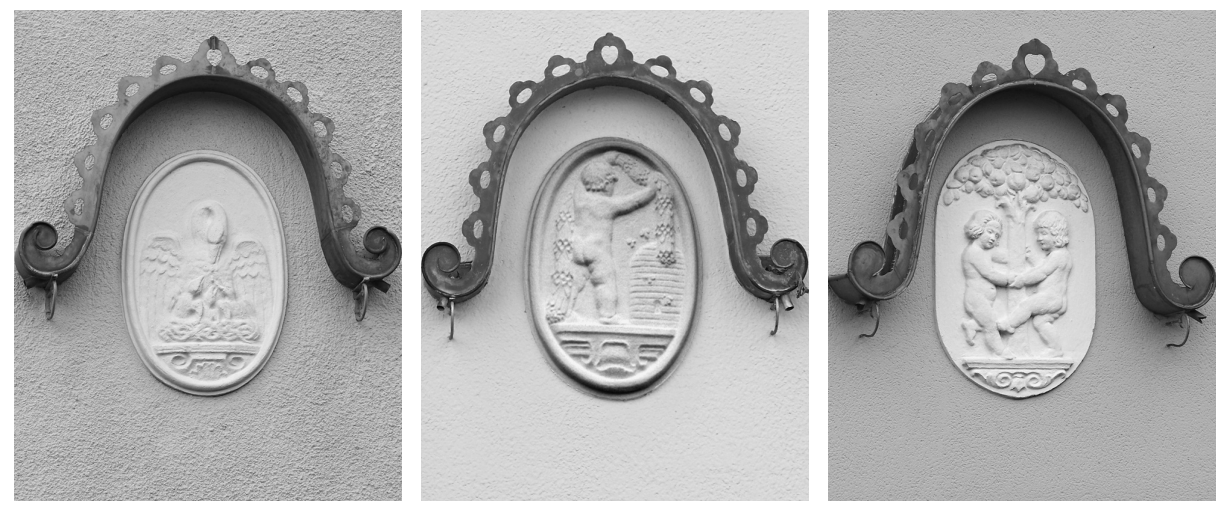

Abb. 16-18: Kolonie Marga, Pelikan-Medaillon (L2-Ringstraße/Nordstraße), Bienenkorb-Medaillon (L3 - Ernst-Thälmann-Straße), Tanz-Medaillon (L5 - Ringstraße/Kirchstraße).

In zeitgenössischen Quellen wird die Kolonie Marga häufig als im „niedersächsischen Stile" erbaut beschrieben. ${ }^{35}$ Diese Formulierung scheint auf den ersten Blick zwar fremd, aber nicht gänzlich abwegig, assoziiert man doch mit ihr Architekturmotive wie Erker, Ausluchten, Fachwerk, einen lebendigen Wechsel von Traufund Giebelständigkeit und anderes mehr und genau diese Motive finden sich in Marga in verschwenderischer Fülle. Doch widersprechen diese, durch den fehlenden Regionalbezug, nicht eigentlich den Intentionen traditionalistischer Architektur, die im Anknüpfen an regionale Bautraditionen eine Rückbesinnung auf eine

34 KRÜGER, Verhältnisse in der Braunkohlenindustrie (wie Anm. 5), S. 113.

35 Führer, hrsg. von der Ilse-Bergbau-Actiengesellschaft, Berlin [um 1920], S. 73. 
vermeintlich gute alte Zeit propagierte? Keineswegs, denn es ist ein Irrglaube, dass der frühe Traditionalismus an regionalen historischen Vorbildern geschult ist. Diese Architektur steht vielmehr am Beginn des Weges zu einem internationalen Stil, zu einer moderaten Moderne. Im konkreten Fall darf auch nicht übersehen werden, dass es in der Niederlausitz kaum praktikable historische Vorbilder gab, denn bis weit in das 19. Jahrhundert war die Region vor allem agrarisch geprägt und wies außerhalb der wenigen Ackerbürgerstädte eine starke slawische Tradition auf, die sich architektonisch in der typischen hölzernen Blockbauweise äußerte. Diese konnte keine modernen Gestaltungsvorbilder liefern, weder aus praktischen Erwägungen noch aus ideologischen Gründen. In diesem Sinn verwendbar war allenfalls die herrschaftliche Architektur des Niederlausitzer Landadels. Diese von sächsisch-barocken Einflüssen geprägte Schlossarchitektur wurde in Marga um eine bäuerlich-sächsische Wohnhausarchitektur, wie sie der traditionelle Dreiseithof darstellt, angereichert und durch Motive der modernen englischen Landhaus-Architektur ergänzt. Doch rechtfertigt dies kaum die Bezeichnung als „modernisierter niedersächsischer Stil“!

Am Ende des 19. Jahrhunderts stand Deutschland in starker Konkurrenz zu den führenden europäischen Wirtschaftsmächten. Englische Waren und Industrieprodukte galten als innovativ und qualitativ hochwertig, deutsche Produkte hingegen als Plagiate und minderwertig in ihrer Qualität, was letztlich zur Stigmatisierung „made in germany“ führte. Auch französische Waren - Frankreich selbst war gerade erst im Krieg durch Deutschland besiegt worden - galten als hochwertig und vor allem als Lifestyle-Produkte. ${ }^{36}$ In bürgerlich konservativen Kreisen formierte sich gegen die ungebrochene kulturelle Überlegenheit Frankreichs wachsender Widerstand, da man den Niedergang deutscher Kultur und Identität befürchtete. Es war das Buch „Rembrandt als Erzieher“, verfasst von Julius Langbehn zwischen 1885 und 1890 in Dresden und veröffentlicht unter dem Pseudonym „Von einem Deutschen“, das diesen Befürchtungen Ausdruck verlieh und das in dem Postulat einer Rückbesinnung auf die historischen und kulturellen Wurzeln der Deutschen ein Konzept zur kulturellen Erneuerung anbot. ${ }^{37} \mathrm{Nach}$ Langbehn sei dem Deutschen nicht die lateinische Ratio, sondern vielmehr eine naturverbundene Emotionalität eigen, zu der es zurückzukehren gelte. Erhalten habe sich eine solche Emotionalität vor allem in der niederdeutschen Region, die weite Teile des heutigen Norddeutschlands und Gebiete in Holland umfasst. In den Künsten, insbesondere aber in der Malerei - nach dem Vorbild Rembrandts -

36 Diese Wahrnehmung, vor allem auf dem deutschen Markt, führte dazu, dass selbst deutsche Produktentwicklungen französische Namen erhielten, um diese aufzuwerten. Ein hervorragendes und besonders kurioses Beispiel stellt das Braunkohlenbrikett dar, ein Produkt, das in Mitteldeutschland entwickelt wurde und das unter der französischen Bezeichnung „Briquette“ (franz. für Ziegelsteinchen) in die deutschen Haushalte geliefert wurde.

37 Zur Geschichte und Bedeutung dieses Buches vgl. Jürgen Paul, Der „Rembrandtdeutsche“ in Dresden, in: Dresdner Hefte 57 (1999), S. 4-13. 
sah Langbehn die künstlerische Leitdisziplin, die zu einer solchen Erneuerung fähig sei. Während andere deutsche Kulturräume ihm als verdorben oder zumindest verbildet galten, glaubte er im rückständigen, agrarisch geprägten niederdeutschen Raum diese naturverbundene Emotionalität noch vorhanden. Der Begriff „Niederdeutsch“ wird so zum Synonym für den bäuerlichen, für den erd- und naturverbundenen Menschen, der - dem griechischen Göttersohn Antaeus gleich - seine schöpferische Kraft in unbegrenztem Ausmaß aus dem heimatlichen Boden ziehen könne..$^{38}$ Aus diesem Grund ist in Marga das ländlich-bäuerliche Motiv allgegenwärtig. Und so wie Langbehn eine Besinnung auf die kulturellen Wurzeln fordert, so schöpft von Mayenburg aus einem heimischen Architekturkanon, den er um Motive der modernen englischen Landhausarchitektur bereichert. Und selbst darin besteht kein Widerspruch zu den Gedanken Langbehns, zählte dieser doch auch den Engländer William Shakespeare zu den herausragenden Vertretern des niederdeutschen Geistes. ${ }^{39}$ Selbst die starke Individualisierung, die die Einzelgebäude durch die reiche Verwendung unterschiedlicher architektonischer Motive und Elemente erfahren haben, lässt sich mit Worten Langbehns legitimieren: Die treibende Grund- und Urkraft des Deutschtums aber heißt: Individualismus. ,Charakter haben und deutsch sein, ist obne Frage gleich bedeutend', sagt Fichte. $Z u$ dieser ibm angeborenen, jedoch im Laufe der Zeit vielfach verlorengegangenen Eigenschaft muß der Deutsche zurückerzogen werden. ${ }^{40}$

Die Kolonie Marga kann demnach als steingewordene Interpretation des Langbehnschen Erziehungskonzeptes angesehen werden, in dessen Mittelpunkt die Rückerziehung des Volkes zu deutschen Tugenden stand. Bekanntlich gehören zu ihnen Ordnung, Fleiß, Pünktlichkeit, Loyalität und Pflichtbewusstsein - alles Eigenschaften, an denen auch die Ilse Bergbau AG als Arbeitgeber größtes Interesse hatte, garantierten diese doch einen kontinuierlichen und störungsfreien Produktionsablauf in Grube und Fabrik. Erziehung durch Vorbild ist der Kern dieses pädagogischen Konzeptes, und das Vorbild suchte man in einer Zeit bzw. in einer Region, die noch vor dem Beginn der Industrialisierung stand. ${ }^{41}$ Als besonders wirkungsvolle Mittel wurden Kunst, Kunsthandwerk und Architektur angesehen, was wohl vor allem daran lag, dass diese Künste allgegenwärtig waren, auch unter der einfachen Bevölkerung. Die Kolonie Marga ist Ausdruck dieser pädagogischen Bemühungen, die auf eine tugendvolle Belegschaft abzielten. In Hinblick auf die regionale Lage der Kolonie in der wendischen Niederlausitz dürfte es hier jedoch nicht allein um eine Rückerziehung auf diese Tugenden, sondern zugleich auch um eine Form der Germanisierung dieses vormals slawischen Siedlungsrau-

Julius Langbehn, Rembrandt als Erzieher, Leipzig 1890, S. 7.

Ebd., S. 158.

40 Ebd., S. 3.

41 Dies sind im Übrigen auch die Wurzeln des Traditionalismus, wie er um die Jahrhundertwende durch Autoren wie Paul Schultze-Naumburg, Paul Mebes u. a. in ganz ähnlicher Weise - in Vorbild und Gegenbild - propagiert wurde. 
mes gegangen sein. Ein Ergebnis, zu dem auch Ulf Jacob, allerdings auf Basis ganz anderer Erkenntnisse und Voraussetzungen, gelangt ist. ${ }^{42}$

Der direkte Vergleich der Kolonie Marga mit der um 1909 und damit nur wenig später durch denselben Architekten geplanten Kolonie Heye III bei Bernsdorf verdeutlicht in besonderer Weise die Bedeutung des Bauherrn als Entscheidungsträger eines solchen Bauvorhabens. Während Marga mit einem gewaltigen Wurfe konstruiert ${ }^{43}$ wurde und als Gesamtkunstwerk mit seinem künstlerischen und ideologischen Überbau fasziniert, handelt es sich bei der Kolonie Heye III um eine kleine, malerisch komponierte Ansiedlung mitten im Wald, die weder in ihrer Größe noch in Hinblick auf die Vollkommenheit der Infrastruktur mit ihrer größeren Schwester konkurrieren konnte (Abb. 19). Deutlich zu erkennen sind auch die wachsende Neigung zur Typisierung der Einzelbauten und die Reduzierung individualisierender Tendenzen und Motive. Im Abweichen beider Projekte voneinander wird deutlich, dass für Form und Gestalt eines solchen Vorhabens nicht in erster Linie der schöpferische Wille des Architekten ausschlaggebend war, sondern dass der gestalterische Anspruch und das Budget des Bauherrn die endgültige Form bestimmten.

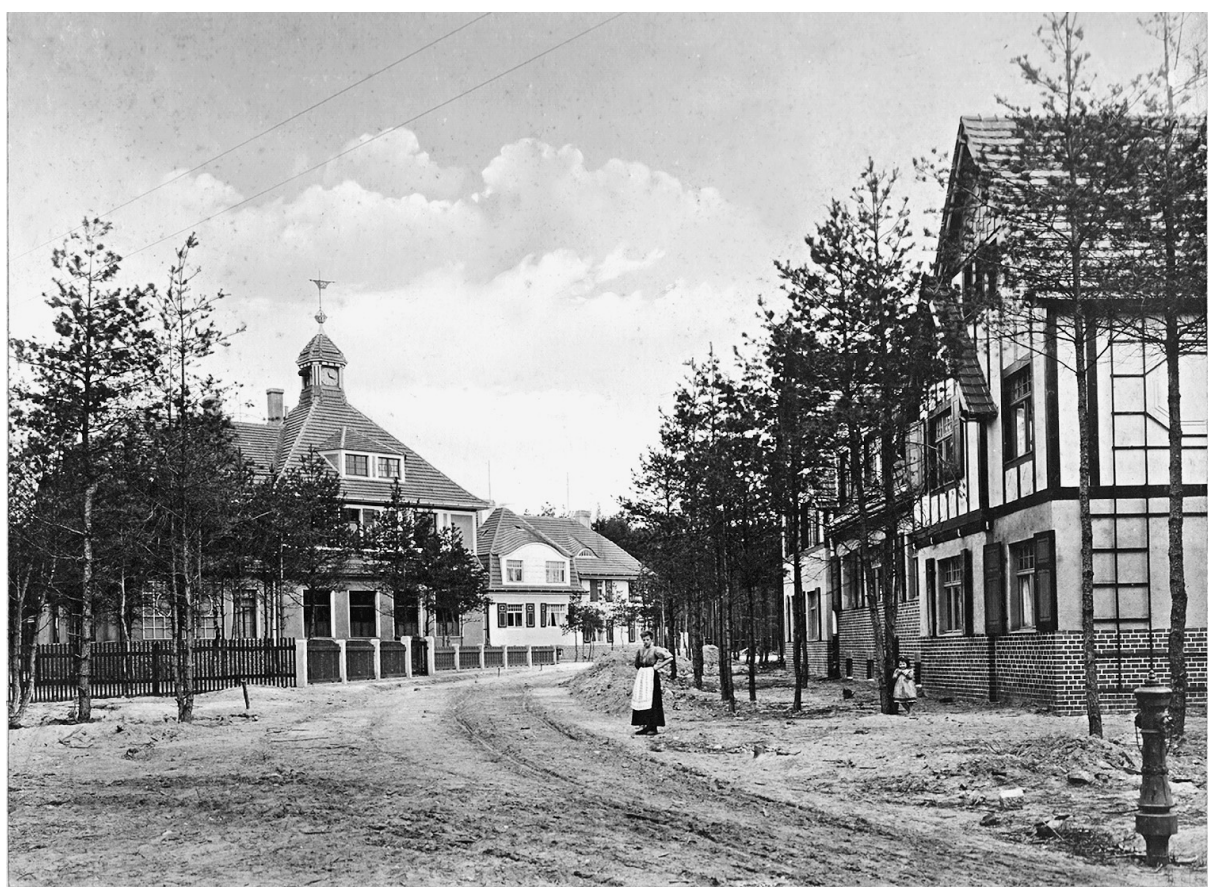

Abb. 19: Kolonie der Grube Heye III, ca. 1915.

42 Jacob/Jochinke, Oasen der Moderne (wie Anm. 9), S. 46.

43 Führer Ilse Bergbau AG (wie Anm. 35), S. 28. 
Zeitgleich zur Realisierung des Großprojektes Kolonie Marga (1906-1914) entstanden zahllose weitere Projekte Georg Heinsius von Mayenburgs. Diese wenigen Jahre müssen als seine produktivsten angesehen werden. Der Ausbruch des Ersten Weltkrieges stellte für ihn wie für zahllose andere Architekten eine wichtige Zäsur dar. Die allgemeine Bautätigkeit kam nahezu zum Erliegen und nur noch kriegswichtige Projekte, wie Rüstungsbetriebe und die dazugehörigen Versorgungseinrichtungen, wurden weiter vorangetrieben. Über die Architektenfirma Klingenberg \& Issel, die 1916/1917 mit der Errichtung des Großkraftwerkes Zschornewitz beauftragt wurde, gelang es auch von Mayenburg als Subunternehmer von einem solchen Projekt zu partizipieren, indem er einen Teil der Bauten der zum Kraftwerk gehörigen Arbeiterkolonie entwarf. Als Offizier der Reserve nahm von Mayenburg aktiv am Ersten Weltkrieg teil. Am Ende des Krieges zog er sich jedoch eine Enzephalitis zu, die ihm die Tätigkeit als Architekt nahezu unmöglich machte. ${ }^{44}$ Aus seiner letzten Lebensdekade sind so auch nur wenige Projekte bekannt, darunter der Umbau von Schloss Eckberg in Dresden, dem Wohnsitz seines Bruders Ottomar Heinsius von Mayenburg..$^{45}$ Es ist jedoch auch denkbar, dass von Mayenburg in dieser Zeit einen Teil seiner verbliebenen Arbeitskraft in die überaus erfolgreichen Werbekampagnen für die ChlorodontProdukte seines Bruders investierte. Das künstlerische Hauptwerk des Architekten, der am 17. April 1930 in Dresden verstarb, ist die Kolonie Marga, die insbesondere durch ihre eigenwillige Melange aus Konservatismus und Modernität besticht.

44 Die Nichte des Architekten - selbst Architektin, jedoch bekannt geworden durch ihre Agententätigkeit für die UdSSR und gegen das NS-Regime in Deutschland - berichtet für die Zeit um 1928 aus dem Hause Georg von Mayenburgs: Onkel Georg wurde kopfgrippekrank, [...] und mein Onkel siechte dabin, im Rollstubl sitzend, mit dem Geiferlätzchen unter dem brabbelnden Mund und darüber bellwache, entsetzlich unglückliche Augen. Er wurde nach Jabren - der Fama nach - von seinem eigenen Arzt umgebracht; Ruth von Mayenburg, Blaues Blut und rote Fahnen. Revolutionäres Frauenleben zwischen Wien, Berlin und Moskau, Wien/München/Zürich 1969, S. 63 f. Weitere Projekte waren der Umbau von Schloss Wackerbarth (Radebeul, 1917-1919), Landhaus Lippold (Dresden-Wachwitz, 1919-1921) und der Umbau des „Hauses am Dinglinger Weinberg" (Dresden-Loschwitz, 1919). 